\title{
Binocular single vision achieved by fusion and suppression
}

\author{
HIROSHI ONO, ROBERT ANGUS, and PETER GREGOR \\ York University, 4700 Keele Street, Downsview, Ontario M3J-1P3, Canada
}

\begin{abstract}
The occurrences of fusion and suppression were determined from stereograms which produced two retinal images located at equal distances but in opposite directions from the fovea. Subjects reported whether the dichoptic stimulus appeared single or not, and if single whether it appeared in the center of the visual field. The report of centrality is predicted by the fusion theory of single vision and that of noncentrality by the suppression theory. Experiment 1, with eight subjects, showed that for small disparities perceived singleness was the percept predicted by the fusion theory; for larger disparities, the percepts could sometimes be predicted by the fusion theory and other times by the suppression theory. Experiment 2, with 16 subjects, showed that with larger stimuli the percept predicted by the fusion theory is more likely to occur. Experiment 3, with four subjects, showed that the centrality was reported when the stimuli were presented for $100 \mathrm{msec}$. This result provided support for our interpretation that the centrality reports in Experiments 1 and 2 were not due to fixation error and suppression.
\end{abstract}

When the two images of a stimulus fall on noncorresponding areas of the two retinae, the stimulus is seen as single, if the disparity is not too large. There are two theories to explain this single vision: the suppression theory and the fusion theory. The suppression theory (e.g., Asher, 1953; Fox \& Check, 1966; Hochberg, 1964; Kaufman, 1974; Ogle, 1962) holds that the singleness of vision is achieved by a process of contralateral suppression, in which the visual input of one eye suppresses the input from the other eye-the process thought to occur during binocular rivalry. Thus, according to suppression theory, the percept is effectively monocular for a portion or the whole of the visual field, i.e., the perceived visual direction is specified by one or the other of the monocular inputs. Fusion theory (e.g., Bishop \& Henry, 1971; Boring, 1933; Charnwood, 1954; Dodwell \& Engel, 1963; Julesz, 1971; Sperling, 1970; Werner, 1937), on the other hand, holds that singleness of vision is achieved by a "compromise" between the visual directions signaled by the noncorresponding retinal areas. Thus, according to fusion theory, the perceived visual direction of a stimulus produced by disparate stimulation does not agree with the visual direction produced by either of the monocular images.

This research was supported by Grant A0296 from the National Research Council of Canada. Experiments 1 and 2 are replications of experiments conducted for Gregor's (1973) MA thesis, with some modifications. A portion of Gregor's results is also reported in Ono (1975). The authors wish to thank our many colleagues at York University and Professor Hochberg for their helpful comments on an earlier version of this paper. Requests for reprints should be sent to Hiroshi Ono, Department of Psychology, York University, Downsview, Ontario M3J-1P3, Canada.
Often, the two theories have been considered to be competing theories in explaining single vision (cf. Dodwell, 1970; Hochberg, 1971; Kaufman, 1974). However, the contention of this paper is that they are not competing theories but complementary ones. It is obviously impossible for fusion and suppression to occur simultaneously for a given stimulus, but the two may occur in alternate time periods. Also, fusion may be more likely to occur for some stimuli and suppression for other stimuli. Experimental results supporting either the suppression or fusion theory generally are obtained by presenting a certain binocular stimulus and examining whether suppression or fusion has occurred (e.g., Asher, 1953; Linschoten, 1956). Apparently conflicting evidence can be explained if it is the case that in certain stimulus conditions suppression is more likely to occur than fusion, or vice vesa. Thus, if the present view is correct, the usual either/or approach should be abandoned, and one should examine the experimental conditions under which fusion or suppression is associated with single vision.

The aim of this study was to show that the predictions from the fusion theory and those from the suppression theory hold under different stimulus conditions, i.e., fusion occurs under some conditions and suppression under others. The traditional definitions of the terms "fusion" and "suppression" (e.g., Asher, 1953; Linschoten, 1956) are used in this paper; the distinction between the two was made from subjects' reports of the visual direction of the perceived stimuli. The present experiment investigated how two variables, the extent of binocular disparity and the size of the disparate stimuli, are related to the relative occurrences of fusion and 
suppression. Binocular disparity was varied in Experiment 1 and stimulus size was varied in Experiment 2 . In both experiments, the relative length of time of the occurrences of fusion, suppression, and diplopia were determined while controlling for fixation disparity. To control for fixation disparity, a nonius alignment check was made; the procedure was similar to one used by Ogle, Martens, and Dyer (1967, p. 27) for measuring fixation disparity. Experiment 3 was conducted with additional control to provide further support for our claim that the fusion which occurred in Experiments 1 and 2 was not an artifact of eye movements during stimulus presentations. Care was taken to control for eye position because of the claim that all evidence which is thought to demonstrate fusion can be explained by the suppression theory and fixation disparity (Kaufman, 1974; Pitblado, 1966).'

\section{EXPERIMENT 1}

The occurrence of fusion or suppression can be inferred from the percept reported by a subject when he is presented with stereoscopically dichoptic stimuli which are equally displaced in opposite directions from the central position. If a subject reports a single percept which is noncentral, an occurrence of suppression can be inferred, since the apparent direction of the dichoptic stimulus corresponds to one of the two visual inputs. However, if the subjects reports a single percept which is central, an occurrence of fusion can be inferred, because the apparent direction of the dichoptic stimulus is a compromise between the two visual directions. Essentially, the above is the logic for Asher's stereograms (Asher, 1953), from which he obtained results supporting the suppression theory. In Experiment 1, several modified versions of Asher's stereograms with different amounts of disparity were presented. Subjects were asked to report whether the dichoptic stimulus appeared central, noncentral, or double. The occurrences of both central and noncentral percepts would imply that both fusion and suppression were associated with single vision.

\section{Method}

Stimuli and Apparatus. Each field of the stereograms consisted of a black disk surrounded by a black circle on a white background. There were three sets of stereograms, one set of experimental stereograms and two sets of controls. Each stereogram was made by first drawing it on a large sheet of paper and then photographically reducing it. When the stereograms were viewed with the stereoscope used in the experiment, the inner disk subtended $2^{\circ}$ of visual angle and the outer circle $6^{\circ}$. The fixation stimuli were vertical lines placed in either the upper or lower half of small circles located on the top of the outer circles. The vertical line in one field was located in the top half of the small circle, and the vertical line in the other field was located in the bottom half of the small circle. When the two lines were seen as falling on a vertical line (nonius alignment), the fixation or convergence response was appropriate for the experiment, i.e., the large outer circles fell on corresponding points and the inner disk produced the disparity intended by the experimenters.

The experimental stereograms were constructed so that, with proper fixation, the images of the inner disks fell at an equal distance, but in opposite directions from the mid-vertical axes of the foveae. There were six experimental stereograms which could be presented either with crossed or uncrossed disparities of $15^{\prime}, 30^{\prime}, 45^{\prime}, 1^{\circ}, 1^{\circ} 15^{\prime}$, and $2^{\circ}$. The same pairs of stimuli were used for presentation of crossed and uncrossed disparities by reversing the relation between stimuli and the eyes. An example of the stereograms from the experimental set and the four possible percepts for each stereogram are shown in Figure 1. The inputs to the left and right eyes are shown as A and B, respectively. With correct fixation, there are four possible percepts, C, D, E, and F. From percept $C$, in which the perceived location of the inner disk is in the center of the outer circle, the occurrence of fusion is inferred. From percepts $D$ and $E$, in which the perceived locations of the disk correspond to the input of one eye, suppression is inferred. Percept F, in which two disks are perceived, is diplopia.

One of the two sets of control stereograms was constructed with no disparity between the monocular inputs; there were three stereograms in this set. For one stereogram, the disks were located in the center of the outer circle. This pair of stimuli was used to determine if subjects would report centrality when the disks were centrally located. For the two other stereograms, the disks were not located in the centers. In one, the two monocular disks were displaced so that the disks fell 7.5' to the left of the foveal midline, and in the other, $7.5^{\prime}$ to the right. These two stereograms were used to determine whether subjects would report noncentrality of the perceived disk when the monocular disks were, in fact, imaged off center. The 7.5' displacement corresponded to the smallest displacement in the experimental stereograms, i.e., for the stereogram with $15^{\prime}$ disparity, the disks were displaced 7.5' from the center in opposite horizontal directions. The reason for determining whether subjects can discriminate $7.5^{\prime}$ displacement of disks from the nondisplaced disks was to determine
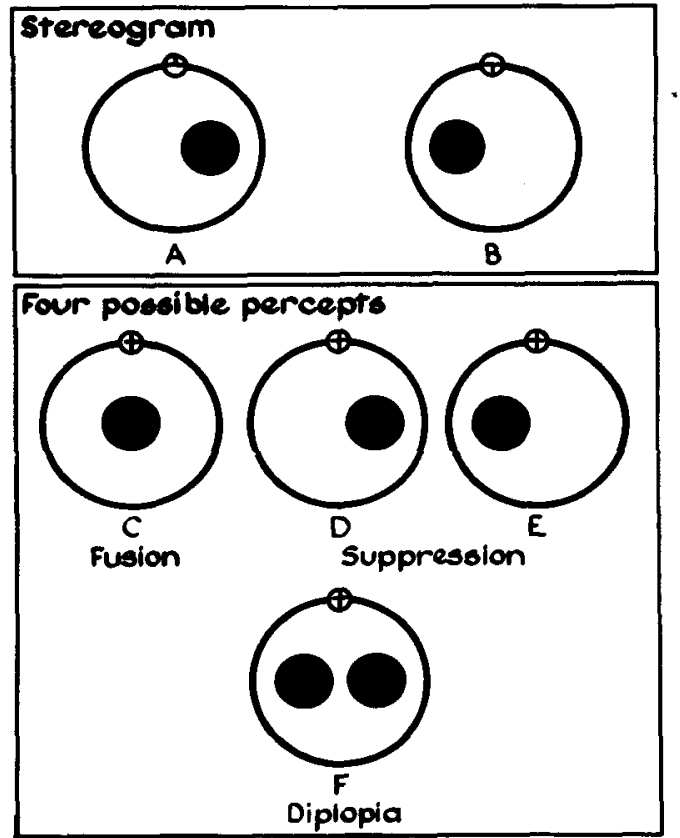

Figure 1. An example of the experimental stereograms used in Experiment 1 and the four possible percepts from the stereogram. 
whether subjects could make this discrimination; if they could, we can distinguish between the occurrence of fusion and suppression for experimental stereograms with $15^{\prime}$ disparity.

The second set of control stereograms, like the experimental stereograms, was constructed so that the inner disks of each pair produced disparity. Unlike the experimental stereograms, however, the disks were not equally displaced from the midline. They were constructed so that, if fusion occurred, a single disk would be perceived either $7.5^{\prime}$ to the left or $7.5^{\prime}$ to the right of center. The disparities produced by these stereograms were $15^{\prime}, 30^{\prime}$, $45^{\prime}$ and $1^{\circ}$. There were two stereograms for each disparity, one which would fuse $7.5^{\prime}$ to the left of center, the other $7.5^{\prime}$ to the right. Thus, there were eight stereograms in this set. The reason for presenting these control stereograms was to obviate a possible criticism that reports of centrality with the experimental stereograms were due solely to the difficulty of directional judgments when the disk is perceived in depth. These control stereograms produced the same depths as the experimental stereograms, but the inner disks should be seen as noncentral if fusion occurs. Therefore, if fusion occurs, the respective experimental stereograms should produce longer durations of centrality judgments than these control stereograms.

The stereograms from the three sets were presented in a Keystone Telebinocular apparatus containing a 19.6-cm focal length lens and 6.8-diopter prisms. The stereograms were placed in a stimulus holder at the focal distance of the lens. Lateral heterophoria was corrected by adjusting the lateral position of each field in the stimulus holder.

Four timers, calibrated in tenths of a second and connected to two switch boxes, were used to record subjects' reports of their percepts. One switch box had one button and was used to indicate when the two fixation (nonius) lines appeared to be on a vertical line. The other switch box had three buttons and was used to indicate whether the inner disks appeared central, noncentral, or double (or noncircular). The timers attached to the three buttons recorded the duration of time each of the three buttons was being pressed conditional on the button on the other switch box being pressed, i.e., the subject's report of his percept of the disk was not recorded unless he was also reporting correct fixation.

Procedure. Each subject was instructed to fixate the center of the small fixation circle throughout each trial, and it was emphasized that he should not change the location of his fixation. He was asked to press, continuously, with his left hand, the button on the switch box with one button when the two nonius lines appeared to be on a vertical line and to release the button when the lines were not on a vertical line. Each subject was also asked to categorize his perception of the black disk and report his perception by using the switch box with three buttons. Each button was used to report one of the following three categories: (a) one disk perceived in the center of the outer circle, (b) one disk perceived not in the center, and (c) two disks or a noncircular disk perceived. If perception changed during a stimulus presentation, the subject was to change his response accordingly. To familiarize the subject with the experimental task, a number of stereograms were shown, and the subject operated the switches until he felt ready for the experiment.

There were two blocks of trials; each trial consisted of presenting one of the 17 stereograms, 3 of which had no disparity. In one block, the remaining 14 stereograms had uncrossed disparity, while in the other block, the 14 stereograms had crossed disparity. For half of the subjects, the block which included the stereograms with crossed disparity was given first; and for the other half, the other block was given first. The order of presenting the 17 stereograms within a block was random. Each trial began when the experimenter removed a blind between the stereogram and the subject's eyes, and ended following $30 \mathrm{sec}$ of appropriate fixation, when the blind was replaced. Before the $1 \mathrm{st}, 4 \mathrm{th}$, 9 th, and 14th trials, the three control stereograms with no disparity were shown twice in random order, and the subject was asked to report verbally whether the inner disk appeared in the center or not. In a preliminary study without this procedure, many subjects misjudged the location of the disk. Thus, the procedure was included to set the subject's criterion for what he reported as central and noncentral.

The subject set his own pace in starting each trial. Each block of trials took about $45 \mathrm{~min}$. A 10 -min rest was given between the two blocks.

Subjects. The subjects were eight volunteers from the university community and were paid for their services. They had no known anomaly in either acuity or stereopsis.

\section{Results and Discussion}

The basic data obtained for each stereogram were the proportions of time the inner disks were reported to be central, noncentral, and diplopic, given appropriate fixation. Analyses were performed on the obtained proportions.

First, the results from the control stereograms will be discussed. Results from the first set of control stereograms (no disparity stereograms) showed that subjects could discriminate a $7.5^{\prime}$ displacement of inner disks from no displacement. The mean proportions of centrality reported was .99 when the inner disks were in fact central and .02 when they were not central.

Table 1 contains the mean proportions of centrality reports for the second set of control stereograms and for the corresponding experimental stereograms. The table shows that there were fewer reports of centrality for the control stereograms than for the experimental stereograms. In the table, the results for both the left and right displacements are combined. For the $15^{\prime}, 30^{\prime}$, and $45^{\prime}$ disparity stereograms, the differences in the mean proportions between controls and experimentals were $.89, .55$, and .19 , respectively, which are all statistically significant, $t(7)=15.74$, $\mathrm{p}<.001, \mathrm{t}(7)=12.21, \mathrm{p}<.001$, and $\mathrm{t}(7)=2.71$, $\mathrm{p}<.05$, respectively. For the $1^{\circ}$ disparity stereograms, the difference was .02 , which is not statistically significant. There were corresponding significant differences in opposite directions for the mean proportions of noncentrality reports, but there were no significant differences for the mean proportions of diplopia reports.

The results suggest that fusion occurred. Consider the results of the $15^{\prime}$ stereograms shown in Table 1.

Table 1

Mean Proportions of Centrality Reported for the Second

Set of Control Stereograms and for the Corresponding Experimental Stereograms

\begin{tabular}{ccc}
\hline & \multicolumn{2}{c}{ Proportions } \\
\cline { 2 - 3 } Disparity & $\begin{array}{c}\text { Control } \\
\text { Stereograms }\end{array}$ & $\begin{array}{c}\text { Experimental } \\
\text { Stereograms }\end{array}$ \\
\hline $15^{\prime}$ & .09 & .98 \\
$30^{\prime}$ & .16 & .71 \\
$45^{\prime}$ & .11 & .30 \\
$1^{\circ}$ & .07 & .09 \\
\hline
\end{tabular}

Note-Disks displaced $7.5^{\prime}$ to the left or right of center. 
For the experimental stereograms, if suppression occurred, a single disk would appear $7.5^{\prime}$ off center; on the other hand, if fusion occurred, the disk would appear central. In the control stereograms, one disk was centrally located while the other disk was located $15^{\prime}$ away from center. Thus, if only suppression occurred, one would expect that the proportions of centrality and noncentrality reports would both be equal to approximately .5 , assuming that the input to each eye was alternately suppressing the other input an equal proportion of time. However, the mean proportion of centrality for the control stereograms was only .09 , i.e., the subjects usually perceived the disk off center. Thus, the significant difference showed that the percepts predicted by the fusion theory predominated when the experimental stereograms with $15^{\prime}$ disparity were presented.

For the experimental and control stereograms with $30^{\prime} 45^{\prime}$, and $1^{\circ}$ disparity, the assumption was that there should be no centrality reports if only suppression occurred. If fusion and suppression take place alternately, there should be some centrality reports for these three experimental stereograms but there should be no such reports for the corresponding control stereograms. However, the control stereograms produced relatively high proportions of centrality reports. The high proportions may be due to either one of two possibilities. One is that the perceived depth of the disk created by the disparities made the judgment of centrality difficult when fusion occurred. The second is that the apparent visual direction of the inner disk when fusion occurred was not exactly the outcome of the arithmetic average of two local signs as traditionally assumed, i.e., local sign values from one eye may be weighted more by the visual system. Whatever the reason, however, it is safe to infer the fusion had occurred. Otherwise, the greater proportion of centrality reported for the experimental stereograms cannot be explained, albeit one of the differences was not statistically significant. Furthermore, the expected extent of noncentrality when suppression occurred for the experimental stereograms was much greater than the expected extent of noncentrality when fusion occurred for the control stereograms, e.g., for the $1^{\circ}$ experimental stereograms, the extent of noncentrality should be $30^{\prime}$ if suppression occurred, whereas for the control stereograms the extent should be 7.5' if fusion occurred. The most conservative interpretation of the results is that the observed proportions of centrality did not exactly reflect the fusion that did take place and that they are only a rough estimate. In any case, it is difficult to argue that fusion did not occur when the experimental stereograms were presented.

For the results from the experimental stereograms, analyses of variance were performed on the three de- pendent variables to determine the effects of the sign of the disparity (crossed vs. uncrossed) and the magnitude of disparity $\left(15^{\prime}\right.$ through $\left.2^{\circ}\right)$. The effect of disparity signs was not significant for any of the reports. Effects related to the magnitude of disparity, the variable of primary interest in this experiment, were statistically significant; for centrality, $F(5,42)$ $=152.96, \mathrm{p}<.01$; for noncentrality, $F(5,42)=8.84$, $p<.01$; and for diplopia, $F(5,42)=28.76, p<.01$. The assumption of equal covariance for a repeatedmeasurements design was not tested, but the GeisserGreenhouse univariate conservative $F$ test indicated significant main effects for all dependent variables. The interactions were not significant.

Figure 2 illustrates the effects of the different extents of disparity on the mean porportions of time the three reports were given. The mean proportions shown were obtained by averaging across two disparity signs and across eight subjects. The graph includes the mean proportions for the no-disparity control stereogram with centrally located disks. The figure shows that the occurrence of fusion, indicated by the mean proportions of the centrality reports, decreased as disparity increased. The occurrence of suppression, indicated by the mean proportions of noncentrality reports, increased up to $45^{\prime}$ and remained approximately constant up to $1^{\circ} 15^{\prime}$. Diplopia began before $30^{\prime}$ and continued to increase. The apparent gradual changes in the three percepts as a function of the magnitude of disparity shown in Figure 2 are partly due to the plotting of mean proportions. An examination of each subject's data showed that some subjects changed abruptly from one percept to another, e.g., a particular subject gave only centrality reports for $15^{\prime}$ and $30^{\prime}$ disparities

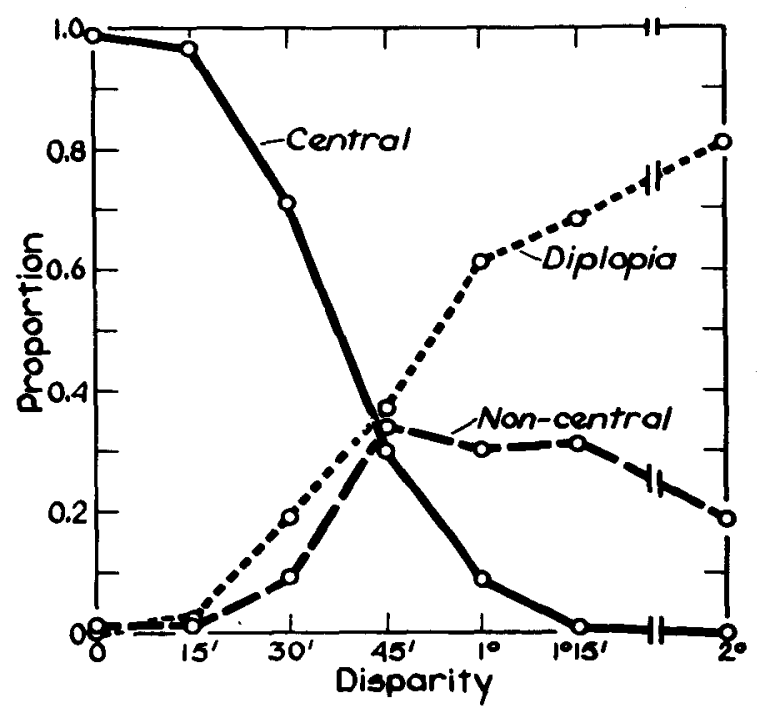

Figure 2. The proportions of centrality, noncentrality, and diplopia reports as a function of disparity. 
but gave no centrality reports for disparities greater than $45^{\prime}$.

The results from the experimental and control stereograms taken together strongly imply that both fusion and suppression occur to achieve single vision. Perceived singleness of vision predicted by the fusion theory held when disparity was small. When disparity was larger, the predictions from both the fusion theory and the suppression theory held. Thus, these results support our contention that the perceived singleness of vision is not limited to the percept predicted by either fusion theory or suppression theory alone.

A brief comment will be made concerning the extent of single vision obtained in Experiment 1. The extent of "Panum's area" generally stated is considerably smaller than the extent of single vision obtained in this experiment. The horizontal extent of Panum's area is considered to be in the range of $9^{\prime}-15^{\prime}$ (cf. Mitchell, 1966). The present results, however, indicate that the extent would be somewhere between $45^{\prime}$ and $1^{\circ}$, if the proportion of .5 of single vision (nondiplopia) is used as the criterion for the extent. The larger area found in the present experiment may be due to the size of the stimulus used, since smaller stimuli are generally used to map Panum's area. This implies that the extent of single vision is a function of stimulus size. Experiment 2 investigates this implication.

\section{EXPERIMENT 2}

The aim of Experiment 2 was the same as that of Experiment 1, namely, to show that single vision is achieved by the perceptual outcomes predicted by both fusion and suppression theories. In Experiment 2 , the size of the disparate stimuli was varied with different sets of stereograms. The basic idea for the stereograms used and the dependent variable were the same as those of Experiment 1, but the stereograms for Experiment 2 were made to allow for a greater range of sizes of the inner disk.

\section{Method}

The apparatus, most aspects of the stimuli, and the general procedure were the same as those of Experiment 1. Only the differences from Experiment 1 will be described in this section.

Stimuli. The inner disks varied in size and were surrounded by letters instead of a circle. The periphery of the stereograms was similar to those of Ogle et al. (1967). According to Ogle et al. (1967), the image of the extrafoveal letters aid the maintenance of motor fusion (as opposed to perceptual fusion). The letters were arranged to permit the presentation of larger disks than were used in Experiment 1. The apex of the letter, $A$, located on the lower portion of the midline of each field, was meant to serve as a reference for the center of the visual field together with the fixation stimuli. A reproduction of one field of a stereogram is shown in Figure 3.

The experimental stereograms were made so that the inner disks subtended visual angles of $7.5^{\prime}, 15^{\prime}, 30^{\prime}, 1^{\circ}, 2^{\circ}$, and $4^{\circ}$. For each

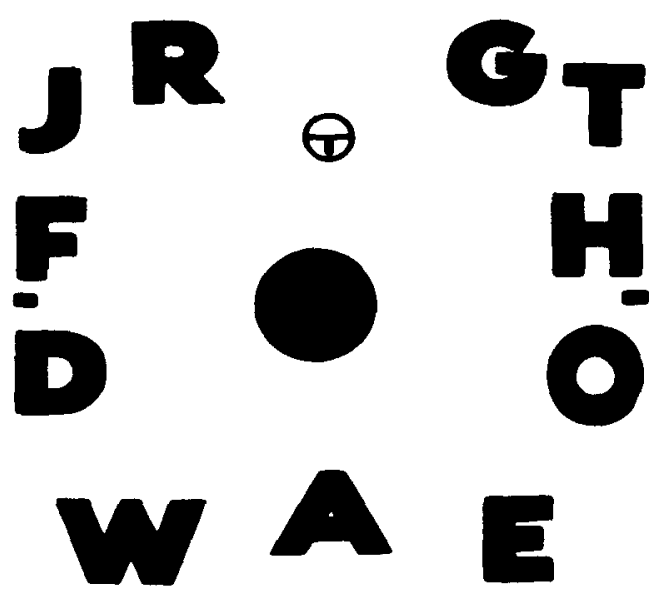

Figure 3. An example of one field of the stereograms used in Experiment 2. The disk of this particular stimulus presented with a stereoscope subtended a visual angle of $2^{\circ}$.

size of disk, two pairs of stereograms were used; one pair produced a horizontal disparity of $30^{\prime}$, and the other produced a disparity of $1^{\circ}$. For each pair, the disks were displaced equal distances but in opposite directions from the midline. Each pair of stimuli for a stereogram was used to present either crossed or uncrossed disparity by reversing the relations between the eye and the stimuli. The rationale of the experimental stereograms was the same as that of Experiment 1 . If fusion occurs, a single disk should be seen in the middle of the visual field; if suppression occurs, a single disk should be seen off center. The $30^{\prime}$ and $1^{\circ}$ disparities were chosen with the expectation that there would be a relatively high proportion of centrality reports for the stereograms with $30^{\prime}$ disparity and a relatively high proportion of noncentrality reports for the stereograms with $1^{\circ}$ disparity.

The set of control stereograms was not as complete, or as elaborate, as those of Experiment 1 for two reasons. One was that the comparison of results from the experimental and control stereograms in Experiment 1 indicated that inferences can be made adequately from the results of the experimental stereograms. The second was that the discrimination of centrality and noncentrality was not as demanding as for some of the stereograms in Experiment 1 . In Experiment 1, the smallest disparity used was 15', which required detecting apparent noncentrality of $7.5^{\prime}$ when suppression occurred, but the smallest disparity used in Experiment 2 was 30', which required detecting apparent noncentrality of $15^{\prime}$ when suppression occurred. The stereograms with a displaced disk having no disparity were not included. Two no-disparity control stereograms were included to determine if subjects would report centrality if the disks were in fact central. For each, both monocular disks were positioned in the center of each field. Disk size was $15^{\prime}$ in one and $2^{\circ}$ in the other. Also included were four pairs of stimuli from which crossed or uncrossed control stereograms could be made. The idea for these control stereograms was the same as that for the corresponding control stereograms used in Experiment 1, namely, to confirm that subjects' reports of centrality were not due to the difficulty of directional judgments when viewing disparate disks in depth. The two disks provided an asymmetrical disparity of $30^{\prime}$, one disk being $7.5^{\prime}$ away from the center in one direction and the other $22.5^{\prime}$ from the center in the opposite direction. In two pairs, the size of the disks was $15^{\prime}$; in the other two, the size was $2^{\circ}$. For each disk size, if fusion occurred, the apparent location of the single disk would be perceived $15^{\prime}$ to the left of center in one pair and 15' to the right of center in the other pair.

Procedure. The procedure was identical to that of Experiment 1 except for the deletion from the experimental session of the 
complete procedure used to set the subject's criterion of what he reported as central. The procedure was used only at the beginning of the experimental session.

Subjects. The subjects were 16 volunteers from the university community who were paid for their services. They had no known anomaly in either acuity or stereopsis.

\section{Results and Discussion}

For the no-disparity control stereograms with disk sizes of $15^{\prime}$ and $2^{\circ}$, the mean proportions of centrality reports were .90 and .92 , respectively, indicating that centrality reports were given reliably when both monocular disks were actually located in the center. For the other control stereograms with disk sizes of $15^{\prime}$ and $2^{\circ}$ and asymmetrical disparities, the mean proportions of centrality reports were .02 and .03 , respectively. In comparison, the mean proportions of centrality reports were .69 and .82 , respectively, for the corresponding experimental stereograms. The differences between the mean proportions from the experimental and control stereograms were statistically significant at the .01 level, $\mathrm{t}(15)=12.38$ and 15.58 for the stereograms with disk size $15^{\prime}$ and $2^{\circ}$, respectively. The reason for the present control stereograms producing smaller proportions of centrality reports than those of Experiment 1 is not obvious to us. However, the comparison of the mean proportions of the experimental and control stereograms indicated more clearly than those of Experiment 1 that the centrality reports reflected the occurrences of fusion.

For the results from the experimental stereograms, analyses of variance were performed on the three dependent variables to determine the effect of disparity signs (crossed vs. uncrossed), disparity values $\left(30^{\prime}\right.$ and $\left.1^{\circ}\right)$, and the size of disks $\left(7.5^{\prime}\right.$ to $\left.4^{\circ}\right)$. Unlike the results of Experiment 1, the main effects of the sign of the disparity were statistically significant. The significant main effects were those of centrality and diplopia reports, $F(1,15)=6.54(p<.05)$ and $13.13(p<.01)$, respectively. The main effect of noncentrality was not significant, $F(1,15)=.95$. The significant effects were the results of the stereograms with crossed disparity producing a higher mean proportion of centrality reports and a lower mean proportion of diplopia reports. Because these directions of differences are the same as those of Experiment 1, the significant sign effects obtained in Experiment 2 are probably due to the larger sample size used. The main effects of disparity values were consistent with those of Experiment 1 and were also statistically significant at the .01 level, $F(1,15)$ $=155.60,7.57$, and 72.13 , for centrality, noncentrality, and diplopia, respectively. The main effects of size, the variable of primary interest in Experiment 2, were statistically significant; $F(5,75)$ $=8.83(p<.01), 2.69(p<.05)$ and $16.62(p<.01)$ for centrality, noncentrality, and diplopia, re- spectively. The Geisser-Greenhouse univariate conservative test was applied to these $F$ values; the effect for centrality and diplopia remains significant at the .01 level, but the effect for noncentrality is no longer significant. There were no significant interactions among the three factors when the GeisserGreenhouse conservative test was applied.

Figure 4 illustrates the main effect of disk size on the mean proportions of time the three reports were given. The mean proportions shown were obtained by averaging across the two disparity signs, two disparity values, and 16 subjects. The figure shows that the occurrences of fusion increase and the occurrences of diplopia decrease as the disk size increases. The occurrence of suppression on the other hand, remains relatively constant across the range of disk sizes, except for the slight increase for disk sizes of $1^{\circ}$ and $2^{\circ}$. The results clearly show that single vision of binocular disparate stimuli is related to the size of the stimulus.

The examination of the $F$ values and the figure may suggest that stimulus size determines the relative occurrence of fusion but not suppression. However, such a conclusion may be premature. Given a constant shape, the size of the stimulus in terms of specifying the experimental stimulus is a precise variable, but in terms of the critical variables for the visual system, "size" lacks precision, i.e., the visual system may be responding to diameter, area, or amount of contour. In the present experiment, with the variation of the disk size, there were variations of contralateral closeness of contours and the extent of overlap of two disks. Viewing the effect of size in this manner, one should note the slight increase in the noncentrality reports for disk sizes of $1^{\circ}$ and $2^{\circ}$, albeit the main effect was not significant when

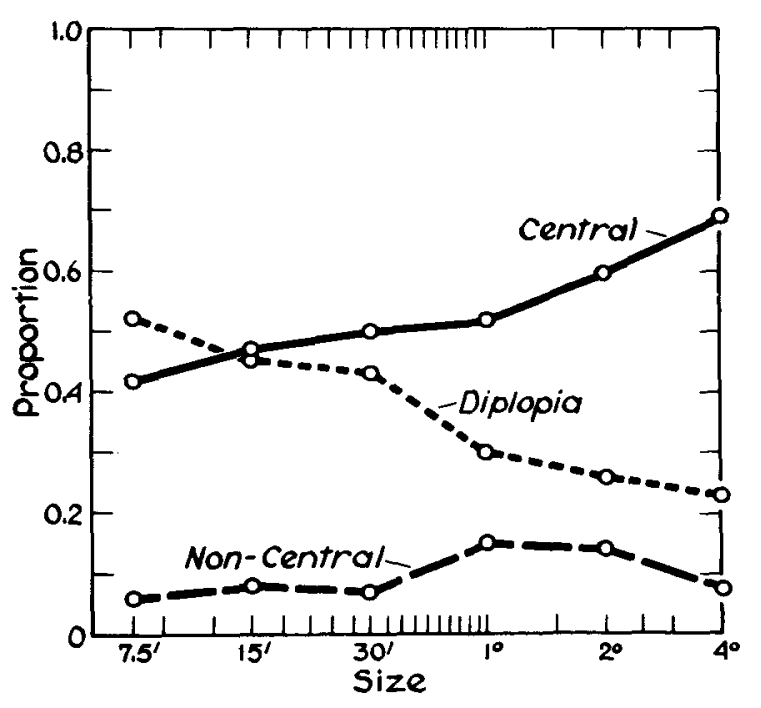

Figure 4. The proportions of centrality, noncentrality, and diplopia reports as a function of size of disparate stimuli plotted on a semilog coordinate. 
the Geisser-Greenhouse conservative $F$ test was applied. Since the disk sizes of $1^{\circ}$ and $2^{\circ}$ contralaterally overlapped at $30^{\prime}$ and $1^{\circ}$ disparity, respectively, the increase may well be related to the contralateral suppression of contours posited by Hochberg (1964) and Kaufman (1963). In fact, among all the $30^{\prime}$ disparity stereograms, the stereograms with $1^{\circ}$ disk size produced the highest mean proportion of noncentrality reports; among all the $1^{\circ}$ disparity stereograms, the stereograms with $2^{\circ}$ disk size produced the highest mean proportion of noncentrality. The implication is that the small effect of size on suppression may be due to the way in which we manipulated the size of the stimulus.

The foregoing discussion places limits on the interpretation of the results of Experiment 2 but does not contradict the contention of this paper, namely, that the percepts predicted by both fusion and suppression theories contribute to single vision. The results of Experiments 1 and 2 should be viewed as the beginning of an attempt to specify some variables which determine when fusion or suppression occurs.

\section{EXPERIMENT 3}

This experiment was suggested to us by Kaufman (Note 1) to strengthen our claim that fusion can occur in achieving single vision; our claim that the results of Experiments 1 and 2 are not confounded by fixation disparity depends on subjects' maintaining fixation as instructed. In Experiment 3, the disparate stimuli were presented for $100 \mathrm{msec}$, when nonius alignment was achieved, to minimize the effects of eye movements during stimulus presentation. As in Experiments 1 and 2, the subjects' task was to judge centrality or noncentrality of the dichoptic stimulus.

\section{Method}

Stimuli and Apparatus. Two sets of red light emitting diodes (LEDs) (Monsanto MV50), each set presented to different eyes, were arranged as shown in Figure 5. (The numbers in the figure are included for descriptive purposes only and were not parts of the stimulus panels.) Different portions of the stimulus panels served different functions. The LEDs in Rows 3 and 7 were used to present the test stimuli, and the LEDs in Column 4 in these rows were also used for fixation stimuli. The LEDs in Rows 1, 2,8 , and 9 were used as reference stimuli for judging whether the test stimuli appeared central or not. The fixation stimuli, which were shown at the beginning of a trial, were the LEDs in Rows 3, 4 , and 5 of Column 4 in one set, and in Rows 5,6 , and 7 of Column 4 in the other set. The reason for this presentation was that when the subject is fixated on the LED in Row 5, correct fixation is indicated for the test stimuli to follow, if the stimuli appeared to be vertically aligned. The stimuli that followed were LEDs in Rows 1, 2, 8, and 9 (reference stimuli) and also the LEDs in Rows 3 to 7 of one of the columns in each set (test stimuli). By presenting different columns in two sets, the necessary disparity was produced.

The stimuli were presented in 10 different configurations. Two configurations were the experimental stimuli; four were the control stimuli and four were used to check the results of the pre-

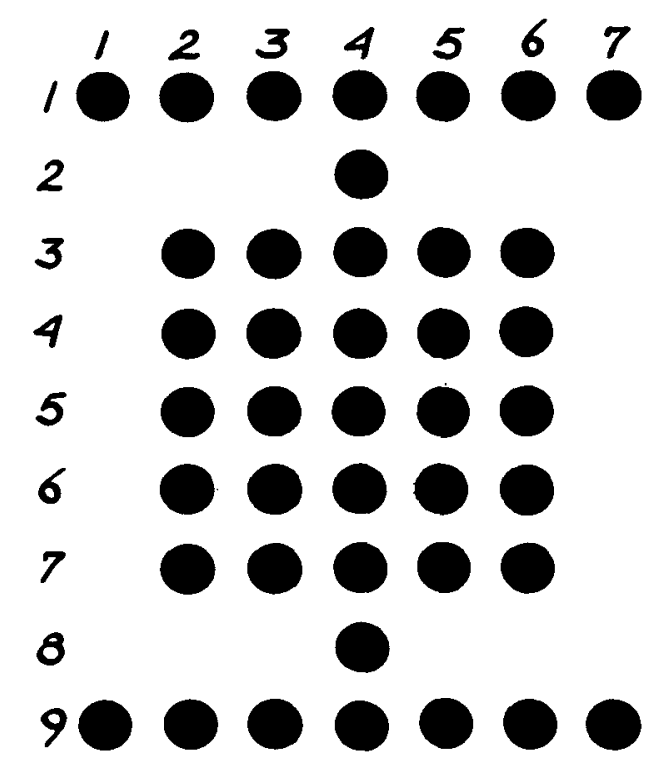

Figure 5. One of the two stimulus arrangements used in Experiment 3.

liminary study for this experiment. (The last four stimuli will be described after the description of the apparatus.) The two experimental and four control configurations are illustrated in Figure 6. The rationale for the configurations depicted in Figure 6 was the same as that for the experimental and control stimuli in Experiments 1 and 2. For the experimental configurations, if fusion occurs the subject should see the stimuli in the center of the "composite" panels (in line with Rows 1, 2, 8, and 9 of Column 4). For the control configurations, if fusion occurs the subject should not see the composite stimuli of these columns in line with Column 4 in the reference stimuli, i.e., the two monocular rows should be seen as single and noncentrally.

The six configurations shown in Figure 6 were produced in the following manner. The two experimental configurations consisted of presenting symmetrical disparity-uncrossed and crossed. For the presentation of uncrossed disparity, Rows 3 to 7 of Column 3 were presented to the left eye and the corresponding rows of Column 5 to the right eye; for the presentation of crossed disparity, the relation between the columns and the eyes was reversed. The control configurations consisted of presenting asymmetrical disparity-two uncrossed and two crossed. For one presentation of uncrossed disparity, Rows 3 to 7 of Column 4 were presented to the left eye and the same rows of Column 6 to the right eye; for the other presentation, Rows 3 to 7 of Column 2 were presented to the left eye and the same rows of Column 4 were presented to the right eye. For the two presentations of crossed disparity, the relation between the eyes and the columns was reversed (see Figure 6).

In the two panels, the actual distances between rows and between columns were $4.3 \mathrm{~mm}$; the panels were viewed through a stereoscope comprised of two identical optical systems. In each system, a variable prism was located near the eye, and a negative 6-diopter lens was placed between the prism and the panel. The lens was located $61.8 \mathrm{~cm}$ and the panel $111.8 \mathrm{~cm}$ from the subject's eye. This optical system produced a virtual image of the display at $74.3 \mathrm{~cm}$ with a virtual separation between the rows and between the columns of $1.08 \mathrm{~mm}$. The visual angles subtended by the virtual separation were $S^{\prime}$. Hence, the extent of disparity produced by each of the six test stimuli was $10^{\prime}$.

The intensity of the LEDs was reduced until no scattered light was seen. The only visible stimuli during the experiment were the lights on the panels. 


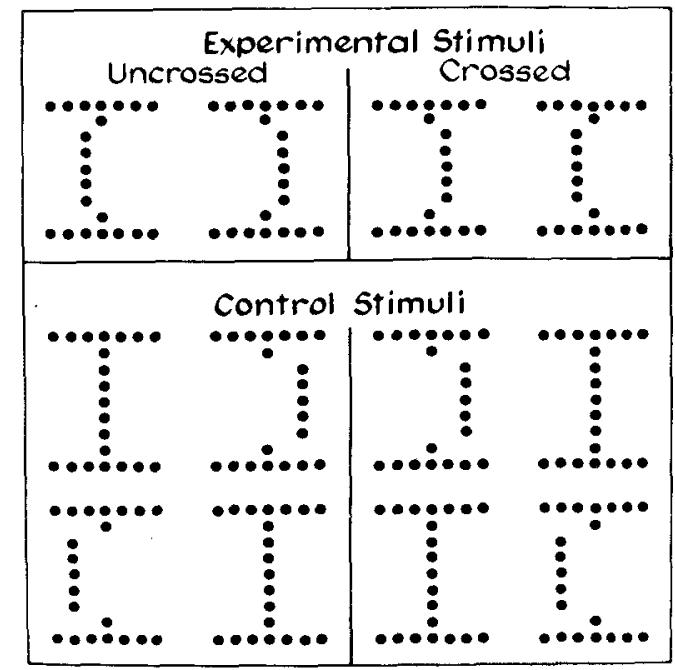

Figure 6. Illustrations of experimental and control stimuli used in Experiment 3.

The choice of a $10^{\prime}$ disparity was based on the results of a preliminary study. This disparity was small enough to produce single vision but large enough to produce noncentral reports if fusion did not occur. The results of the preliminary study indicated that for $10^{\prime}$ of disparity there were very few percepts of diplopia when the stimulus was presented for $100 \mathrm{msec}$. Furthermore, when Rows 3 to 7 of Column 3 or 5 were presented monocularly, with a binocular presentation on Rows 1, 2, 8, and 9, the stimulus was reliably reported to appear noncentral, but when Rows 3 to 7 of Column 4 were presented monocularly with the same binocular stimuli, the stimulus was reliably reported to appear central.

Because the experimental subjects differed from those used in the preliminary study, four monocular test stimuli were presented in the experiment to ascertain whether the $10^{\prime}$ disparity was adequate for the experimental subjects. The first two of the four presentations consisted of central monocular stimuli; the two other presentations consisted of noncentral monocular stimuli. For the central monocular test stimuli, Rows 3 to 7 of Column 4 were presented to one eye and Rows $1,2,8$, and 9 to both eyes. Although there were four possible monocular noncentral test stimuli, two of them were chosen so that there would be an equal number of central and noncentral presentations. The two chosen consisted of presenting (1) Rows 3 to 7 of Column 3 to the right eye and Rows 1, 2, 8, and 9 to both eyes, and (2) Rows 3 to 7 of Column 5 to the left eye and Rows $1,2,8$, and 9 to both eyes.

Procedure. The variable diopter prism was adjusted so that the subject would converge to $74.3 \mathrm{~cm}$. Further adjustment of the apparatus was made for each subject by varying the distance between the two LED panels. The second adjustment was made to eliminate fixation disparity; this was done so that the fixation stimulus was readily seen on a vertical line.

Each trial consisted of three parts: (1) fixation, (2) stimulus presentation, and (3) report of percept. Each subject was asked to fixate on the middle of the "composite" fixation stimuli (Row 5 and Column 4) and to push a switch when the five lights were seen on a vertical line. Pushing the switch turned off the fixation stimuli and turned on one of the combinations of reference stimuli and test stimuli for $100 \mathrm{msec}$. The subject's task was to report whether the test stimuli appeared central, noncentral, or double.

There were two kinds of fixation stimuli. One consisted of the upper three stimuli for one panel being presented to the left eye and the lower three stimuli from the other display being presented to the right eye; the other consisted of the opposite combination.
Combining 2 fixation stimuli and 10 test stimuli gave a total of 20 combinations. The presentation of one of the combinations constituted a trial.

The 20 types of trials were repeated five times. The sequence of the trials was random, with the restriction that in each block of 20 trials, different combinations of stimuli were represented once. The subject set his own pace; the trial began when he was ready.

Subjects. Four subject, acquaintances of the experimenters, participated in the experiment. They had no known anomaly in either acuity or stereopsis. Two subjects knew the purpose of the experiment and the details of the stimulus arrangement; the other two were completely naive concerning the purpose and the details.

\section{Results and Discussion}

The central and noncentral monocular test stimuli were discriminable for the experimental subjects just as they were for the subjects in the preliminary study. The mean proportion of trials in which the central stimuli appeared central was .91 , whereas the mean proportion of trials in which the noncentral check stimuli appeared central was .09. The difference confirmed the results of the preliminary study and indicated that the value of disparity used for the test stimuli was adequate for inferring whether fusion had occurred or not.

The results from presenting the binocular test stimuli indicated that fusion occurred. The mean proportion of trials in which the symmetrical test stimuli appeared central was .88 , whereas the mean proportion of trials in which the asymmetrical test stimuli appeared central was .10. The difference was statistically significant, $\mathrm{t}(3)=18.92, \mathrm{p}<.001$. This significant difference lends credence to our interpretation of the results of Experiments 1 and 2, that the centrality report for the symmetrically disparate stimuli reflects the occurrence of fusion and not fixation disparity and suppression.

Although the present results, in terms of subjects' reporting centrality and noncentrality, agree well with the results obtained when the $15^{\prime}$ disparate stimuli were presented in Experiment 1, there was one major difference in what subjects saw. In Experiment 1, many subjects reported spontaneously that they saw depth between the inner disk and the outer ring, but in Experiment 3, no subject reported seeing depth between the test stimuli and the reference stimuli. We gave extra trials asking for reports of depth judgments, but the subjects did not see depth. The lack of depth perception may be due to different criteria used by two sets of subjects, but was more likely due to the short exposure time and the intensity used. When the test stimuli were exposed for a longer period, the subjects saw depththe crossed stimuli as being closer than the reference stimuli and the uncrossed ones as being farther. One possible implication of the present results is that fusion, defined in terms of the visual system combining two visual directions signaled from two eyes, 
takes place before, or independently of, the processing of depth information.

\section{GENERAL DISCUSSION}

The results of the three experiments demonstrated that perceptual outcomes predicted by both fusion and suppression theories are involved in binocular single vision. Experiment 1 showed that the perceived singleness of dichoptic stimuli with small disparity was the percept predicted by the fusion theory. It also showed that the singleness of the stimuli with larger disparity was sometimes the percept predicted by the fusion theory and other times that predicted by the suppression theory. Experiment 2 showed that the percept predicted by the fusion theory is more likely for a stimulus of larger size. Experiment 3 provided further support for our interpretation of the results of Experiments 1 and 2 that fusion occurs to achieve single vision. The conclusion to be drawn from the results is that the hitherto accepted "either/or" approach, fusion theory vs. suppression theory, should be abandoned. Rather, one should investigate stimulus variables or conditions in which fusion or suppression tend to occur when single vision is achieved.

Although the experiments have shown that single vision is achieved by both fusion and suppression, the conclusion should not be made that disparity and size, the variables investigated, are the only variables that are related to single vision. Other known variables are state of convergence (Richards, 1971; Ronchi, Viliani, \& Barca, 1972), luminance and color (Mitchell, 1966), clearness of contour (Levelt, 1965), spatiotemporal history of stimulation (Fender \& Julesz, 1967), and training (Ames \& Ogle, 1932). Whether and how these variables are related to the occurrences of fusion and suppression are still to be determined, but a complete theory of single vision must specify how these variables and other variables together contribute to single vision.

\section{REFERENCE NOTE}

1. Kaufman, L. Personal communication, 1975.

\section{REFERENCES}

Ames, A., \& Ogle, K. N. Size and shape of ocular images. III: Visual sensitivity to differences in the relative size of the ocular images of the two eyes. Archives of Ophthalmology, 1932, 7, 904-924.

Asher, H. Suppression theory of binocular vision. British Journal of Ophthalmology, 1953, 37, 37-49.

Bishop, P. O., \& Henry, G. H. Spatial vision. Annual Review of Psychology, 1971, 22, 119-160.

BorING, E. The physical dimension of consciousness. New York: Century, 1933. Pp. 118-119.

Charnwood, J. R. B. An answer to the suppression theory. British Orthoptic Journal, 1954, 11, 32-37.

DoDWell, P. C. Visual pattern recognition. New York: Holt, Rinehart, \& Winston, 1970.
Dodwell, P. C., \& ENGel, G. R. A theory of binocular fusion. Nature, 1963, 198, 39-40, 73-74.

Fender, D., \& Julesz, B. Extension of Panum's fusional area in binocularly stabilized vision. Joumal of the Optical Society of America, 1967, 57, 819-830.

Fox, R., \& CHECK, R. Binocular fusion: A test of the suppression theory. Perception \& Psychophysics, 1966, 1, 331-334.

GREGOR, P. Binocular single vision, fusion and suppression: An experimental study. Unpublished Master thesis, York University, 1973.

HoChBERG, J. Contralateral suppressive fields of binocular combination. Psychonomic Science, 1964, 1, 157-158.

Hochbe RG, J. Perception II. Space and movement. In J. W. Kling and L. A. Riggs (Eds.), Woodworth \& Schlosberg's experimental psychology (3rd ed.). New York: Holt, Rinehart, \& Winston, 1971.

Julesz, B. Foundations of cyclopean perception. Chicago: University of Chicago Press, 1971.

KaUFMAN, L. On the spread of suppression and binocular rivalry. Vision Research, 1963, 3, 401-415.

KaUfMan, L. Sight and mind: An introduction to visual perception. New York: Oxford University Press, 1974.

LeVelt, W. J. M. On binocular rivalry. Soesterberg, The Netherlands: Institute for Perception, RVO-TNO, 1965.

Linschoten, J. Strukturanalyse der binokularen Tiefenwahrnehmung. Groningen, Holland: Wolters, 1956.

Mrtchell, D. E. Retinal disparity and diplopia. Vision Research, 1966, 6, 441-451.

OGLE, K. N. Spatial location through binocular vision. In $\mathbf{H}$. Davson (Ed.), The eye (Vol. 4). New York: Academic Press, 1962.

Ogle, K. N., Martens, T. G., \& Dyer, J. A. Oculomotor imbalance in binocular vision and fixation disparity. Philadelphia: Lee \& Febiger, 1967.

ONo, H. Directions of objects seen from the cyclopean eye. Saensu (Japanese edition of Scientific American), 1975, 5, 88-99. (The English translation appeared as Department of Psychology Reports, York University, Toronto, 1976, No. 29.)

Pitblado, C. B. Displacement of half-images during binocular viewing. (Doctoral dissertation, Boston University, 1966). Dissertation Abstracts, 1966, 27, 1641B-1642B. (University Microfilms No. 66-11, 272).

RICHARDS, W. Independence of Panum's near and far limits. American Journal of Optometry, 1971, 48, 103-109.

RonChI, L., Viliani, G., \& BarCa, L. Diplopia and convergence in the neighborhood of absolute threshold. Pubblicazioni Dell'Istituto Nazionale di Ottica, 1972, 27, 765-775.

SPERIING, G. Binocular vision: A physical and neural theory. American Journal of Psychology, 1970, 83, 461-534.

Werner, H. Dynamics in binocular depth perception. Psychological Monographs, 1937, 49(Whole No. 218).

\section{NOTE}

1. In all three experiments, the dependent variable was visual direction, not stereopsis. Subjects reported whether the dichoptic stimuli appeared single or not and, if single, whether it appeared in the center of the visual field. They were not required to report whether or not the stimuli appeared in depth. This fact may be difficult to keep in mind while reading the paper, because the independent variables of the experiments are those usually associated with stereopsis (depth perception). Although the usual discussions of fusion vs. suppression theories are made in the context of the topic of stereopsis, fusion and suppression are not likely to be the necessary or sufficient conditions for stereopsis. Because binocular depth perception occurs with diplopic images, they are not necessary for stereopsis. The results of our Experiment 3 suggest that they are not sufficient.

(Received for publication October 29, 1976; revision accepted February $28,1977$. ) 